\title{
UCRL-TR-218643
}

LAWRENCE LIVERMORE N A T IO N A L LABORATORY
Purple Integration Project

P. Hamilton

February 3, 2006 
This document was prepared as an account of work sponsored by an agency of the United States Government. Neither the United States Government nor the University of California nor any of their employees, makes any warranty, express or implied, or assumes any legal liability or responsibility for the accuracy, completeness, or usefulness of any information, apparatus, product, or process disclosed, or represents that its use would not infringe privately owned rights. Reference herein to any specific commercial product, process, or service by trade name, trademark, manufacturer, or otherwise, does not necessarily constitute or imply its endorsement, recommendation, or favoring by the United States Government or the University of California. The views and opinions of authors expressed herein do not necessarily state or reflect those of the United States Government or the University of California, and shall not be used for advertising or product endorsement purposes.

This work was performed under the auspices of the U.S. Department of Energy by University of California, Lawrence Livermore National Laboratory under Contract W-7405-Eng-48. 
Purple Integration Project

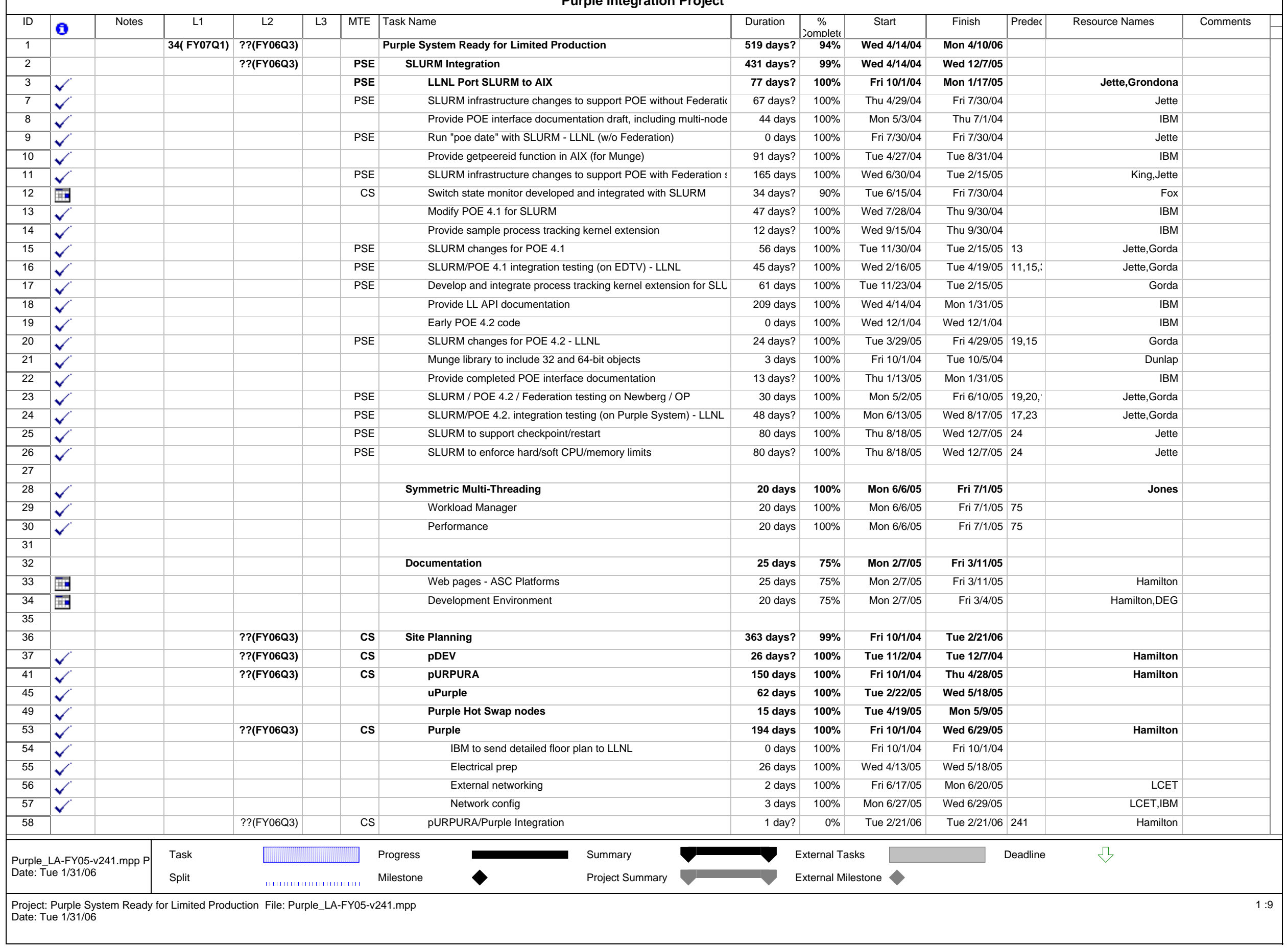




\begin{tabular}{|c|c|c|c|c|c|c|c|c|c|c|c|c|c|c|}
\hline \multicolumn{15}{|c|}{ Purple Integration Project } \\
\hline ID & i & Notes & L1 & L2 & L3 & MTE & Task Name & Duration & $\begin{array}{c}\% \\
\text { :omplett }\end{array}$ & Start & Finish & Predec & Resource Names & Comments \\
\hline 59 & & & & & & & & & & & & & & \\
\hline 60 & $\checkmark$ & & & ??(FY06Q3) & & & pDEV Delivery and Acceptance & 112 days & $100 \%$ & Thu $1 / 6 / 05$ & Fri 6/10/05 & & & \\
\hline 76 & & & & & & & & & & & & & & \\
\hline 77 & $\checkmark$ & & & ??(FY06Q3) & & & pURPURA Delivery and Acceptance & 51 days? & $100 \%$ & Fri 4/15/05 & Fri 6/24/05 & & & \\
\hline 110 & 2 & & & ??(FY06Q3) & & & Integration of pURPURA Complete & 0 days & $100 \%$ & Fri 6/24/05 & Fri 6/24/05 & 577 & & \\
\hline 111 & 2 & & & ??(FY06Q3) & & & Early Access to pURPURA & 1 day? & $100 \%$ & Mon $6 / 27 / 05$ & Mon 6/27/05 & 5110 & & \\
\hline 112 & & & & & & & & & & & & & & \\
\hline 113 & $\checkmark$ & & & & & & uPurple Delivery and Acceptance & 34 days? & $100 \%$ & Mon 5/16/05 & Thu 6/30/05 & & & \\
\hline 147 & & & & & & & & & & & & & & \\
\hline 148 & $\checkmark$ & & & ??(FY06Q3) & & & Purple (1280) System Scale Testing (POK) & 51 days & $100 \%$ & Mon 4/4/05 & Mon 6/13/05 & & & \\
\hline 153 & $\checkmark$ & & & ??(FY06Q3) & & & Purple (1280) Demo/Pre-Ship Test of System in POK & 13 days? & $100 \%$ & Mon 6/13/05 & Thu $6 / 30 / 05$ & & & \\
\hline 156 & & & & & & & & & & & & & & \\
\hline 157 & $\checkmark$ & & & & & & Purple (1280) System Scale Testing (POK) & 35 days & $100 \%$ & Thu $6 / 30 / 05$ & Wed 8/17/05 & 5153 & \multirow{2}{*}{\multicolumn{2}{|c|}{ IBM }} \\
\hline 162 & $\checkmark$ & & & ??(FY06Q3) & & & Purple (1280) System Breakdown & 11 days & $100 \%$ & Thu 8/18/05 & Thu $9 / 1 / 05$ & & & \\
\hline 166 & & & & & & & & & & & & & & \\
\hline 167 & $\checkmark$ & & & & & & Purple at LLNL & 146 days? & $100 \%$ & Thu $4 / 14 / 05$ & Thu $11 / 3 / 05$ & & & \\
\hline 168 & $\checkmark$ & & & & & & Shipping and transit & 122 days? & $100 \%$ & Thu 4/14/05 & Fri 9/30/05 & & & \\
\hline 175 & $\checkmark$ & & & & & & Physical Installation Switch and 252 nodes & 45 days? & $100 \%$ & Tue 5/3/05 & Mon $7 / 4 / 05$ & & & \\
\hline 184 & $\checkmark$ & & & & & & Switch and 252 Node Bringup & 25 days? & $100 \%$ & Thu 6/30/05 & Wed 8/3/05 & & & \\
\hline 190 & $\checkmark$ & & & ??(FY06Q3) & & & Purple (252) Merge with Purple (1024) & 49 days? & $100 \%$ & Mon 8/29/05 & Thu $11 / 3 / 05$ & & & \\
\hline 204 & & & & & & & \multirow[b]{2}{*}{ Purple (1280) Acceptance at LLNL } & & & & & & & \\
\hline 205 & $\checkmark$ & & & ??(FY06Q3) & & & & 11 days & $100 \%$ & Fri 11/4/05 & Fri 11/18/05 & & & \\
\hline 206 & $\checkmark$ & & & & & SE,SS & Test runs and system debug & 4 days & $100 \%$ & Fri $11 / 4 / 05$ & Wed 11/9/05 & 203 & oewe,Fox,Hadjimarkos,IBM & \\
\hline 207 & $\checkmark$ & & & & & $\mathrm{SE}, \mathrm{SS}$ & Acceptance Run (includes Full SWL) & 7 days & $100 \%$ & Thu $11 / 10 / 05$ & Fri $11 / 18 / 05$ & 5206 & oewe,Fox,Hadjimarkos,IBM & \\
\hline 208 & 国 & $\supseteq d$ in the Pur & & ??(FY06Q3) & Yes & & Initial Purple (1280) System Acceptance Complete & 0 days & $0 \%$ & Fri 11/18/05 & Fri 11/18/05 & 5205 & & \\
\hline 209 & & & & & & & & & & & & & & \\
\hline 210 & $\checkmark$ & & & & & & Purple (1280) GA6 Firmware Upgrade & 7 days & $100 \%$ & Mon 11/28/05 & Tue $12 / 6 / 05$ & & & \\
\hline 211 & & & & & & & & & & & & & & \\
\hline 212 & $\checkmark$ & & & & & & Verify application software environmnet & 10 days & $100 \%$ & Wed 12/7/05 & Tue 12/20/05 & 5210 & & \\
\hline 213 & $\checkmark$ & & & & & SE,SS & MPI & 10 days & $100 \%$ & Wed 12/7/05 & Tue 12/20/05 & & Jones & \\
\hline 214 & $\checkmark$ & & & & & & Federation hardening & 10 days & $100 \%$ & Wed $12 / 7 / 05$ & Tue $12 / 20 / 05$ & & & \\
\hline 215 & $\checkmark$ & & & & & & Performance (Co-scheduler, scaling, etc) & 10 days & $100 \%$ & Wed $12 / 7 / 05$ & Tue $12 / 20 / 05$ & & & \\
\hline 216 & $\checkmark$ & & & & & & Tools & 10 days & $100 \%$ & Wed $12 / 7 / 05$ & Tue $12 / 20 / 05$ & & & \\
\hline 217 & $\checkmark$ & & & & & SE,SS & Resource management (LCRM) & 10 days & $100 \%$ & Wed 12/7/05 & Tue $12 / 20 / 05$ & & Tomaschke & \\
\hline 218 & $\checkmark$ & & & & & & Visualization tools & 10 days & $100 \%$ & Wed $12 / 7 / 05$ & Tue $12 / 20 / 05$ & & Springmeyer & \\
\hline 219 & $\checkmark$ & & & & & SE,SS & Debugger & 10 days & $100 \%$ & Wed $12 / 7 / 05$ & Tue $12 / 20 / 05$ & & Wolfe & \\
\hline 220 & $\checkmark$ & & & & & SE,SS & Compilers & 10 days & $100 \%$ & Wed 12/7/05 & Tue $12 / 20 / 05$ & & Gyllenhaal & \\
\hline 221 & $\checkmark$ & & & & & SE,SS & Performance analysis & 10 days & $100 \%$ & Wed $12 / 7 / 05$ & Tue $12 / 20 / 05$ & & Chambreau & \\
\hline 222 & & & & & & & & & & & & & & \\
\hline 223 & $\checkmark$ & & & & & & Science Runs and Early Acess Runs & 20 days & $100 \%$ & Wed 12/7/05 & Tue $1 / 3 / 06$ & 210 & & \\
\hline 224 & & & & & & & & & & & & & & \\
\hline 225 & 国 & & & ??(FY06Q3) & & & Purple Integration into LLNL (SCF) Environment & 8 days & $0 \%$ & Wed 1/4/06 & Fri $1 / 13 / 06$ & 223 & & \\
\hline 226 & & & & & & & Network Configuration & 1 day & $0 \%$ & Wed $1 / 4 / 06$ & Wed $1 / 4 / 06$ & & & \\
\hline Purple & LA-FY05-V & -v241.mpp P & Task & $\square$ & & & Summary & & External Tć & & & Deadline & 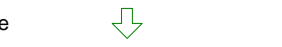 & \\
\hline Date: & ue $1 / 31 / 06$ & & Split & $\mid \ldots \ldots \ldots \ldots \ldots$, & & & Project Summary & & External $\mathrm{M}$ & lestone $>$ & & & & \\
\hline $\begin{array}{l}\text { Projec } \\
\text { Date: }\end{array}$ & $\begin{array}{l}\text { Purple Sy } \\
\text { ue } 1 / 31 / 06\end{array}$ & $\begin{array}{l}\text { ystem Ready fo } \\
6\end{array}$ & r Limited $\mathrm{F}$ & uction File: Pur & ple_LA- & -FY05-v2 & 241.mpp & & & & & & & $2:$ \\
\hline
\end{tabular}


Purple Integration Project

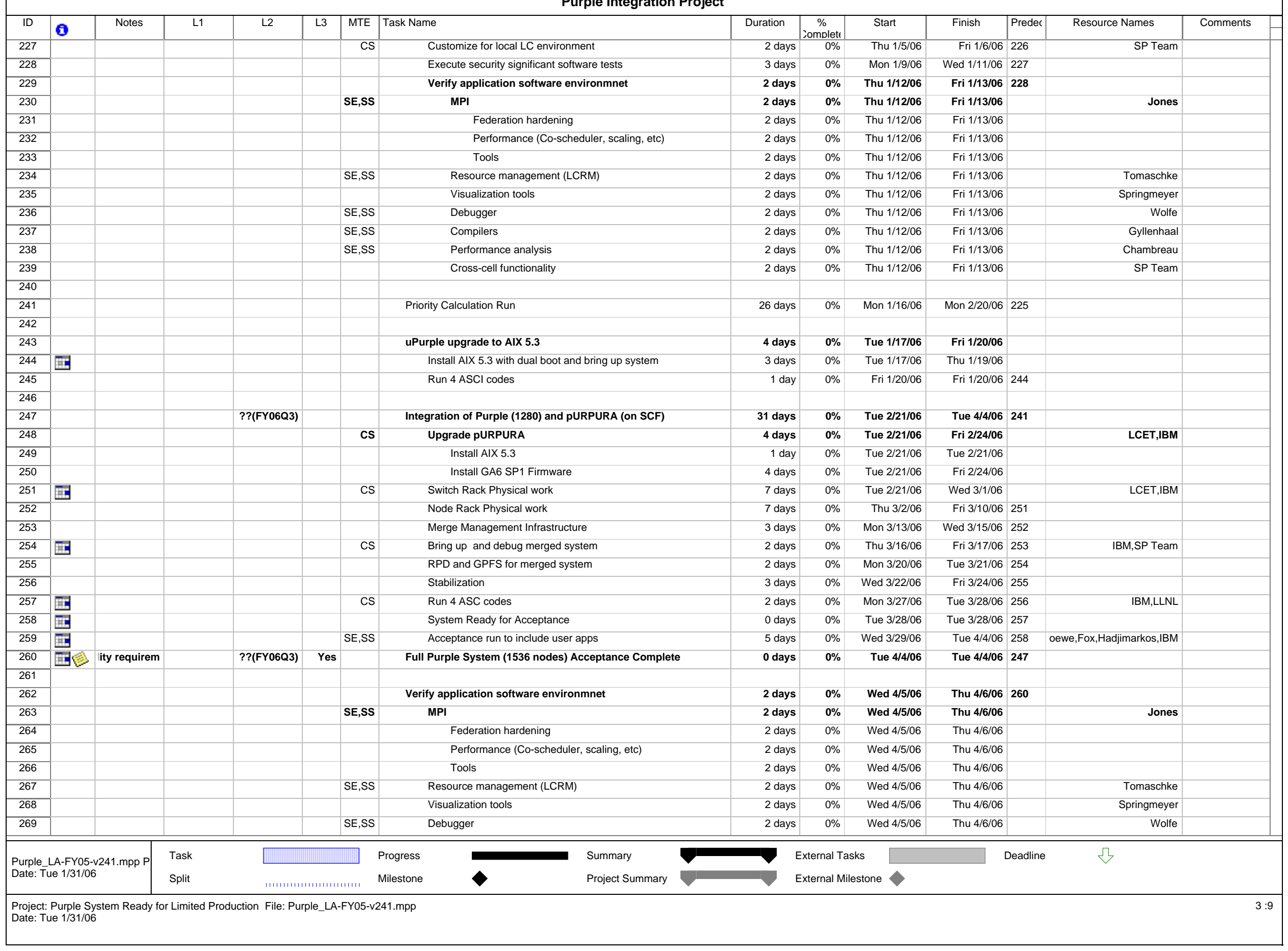




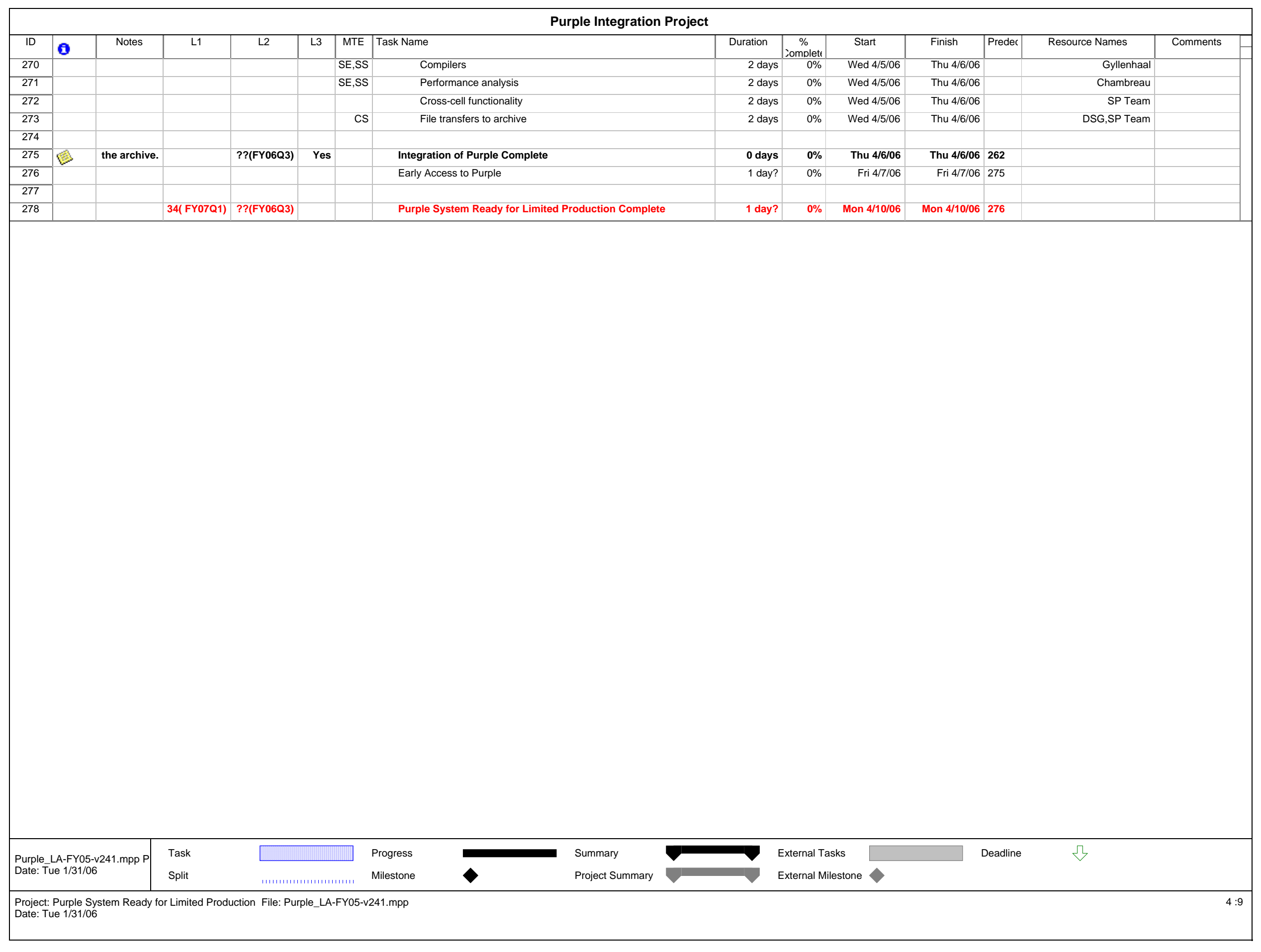


Purple Integration Project

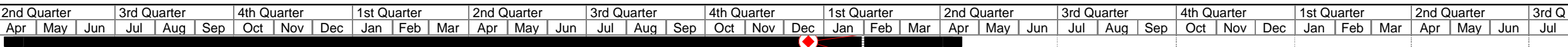

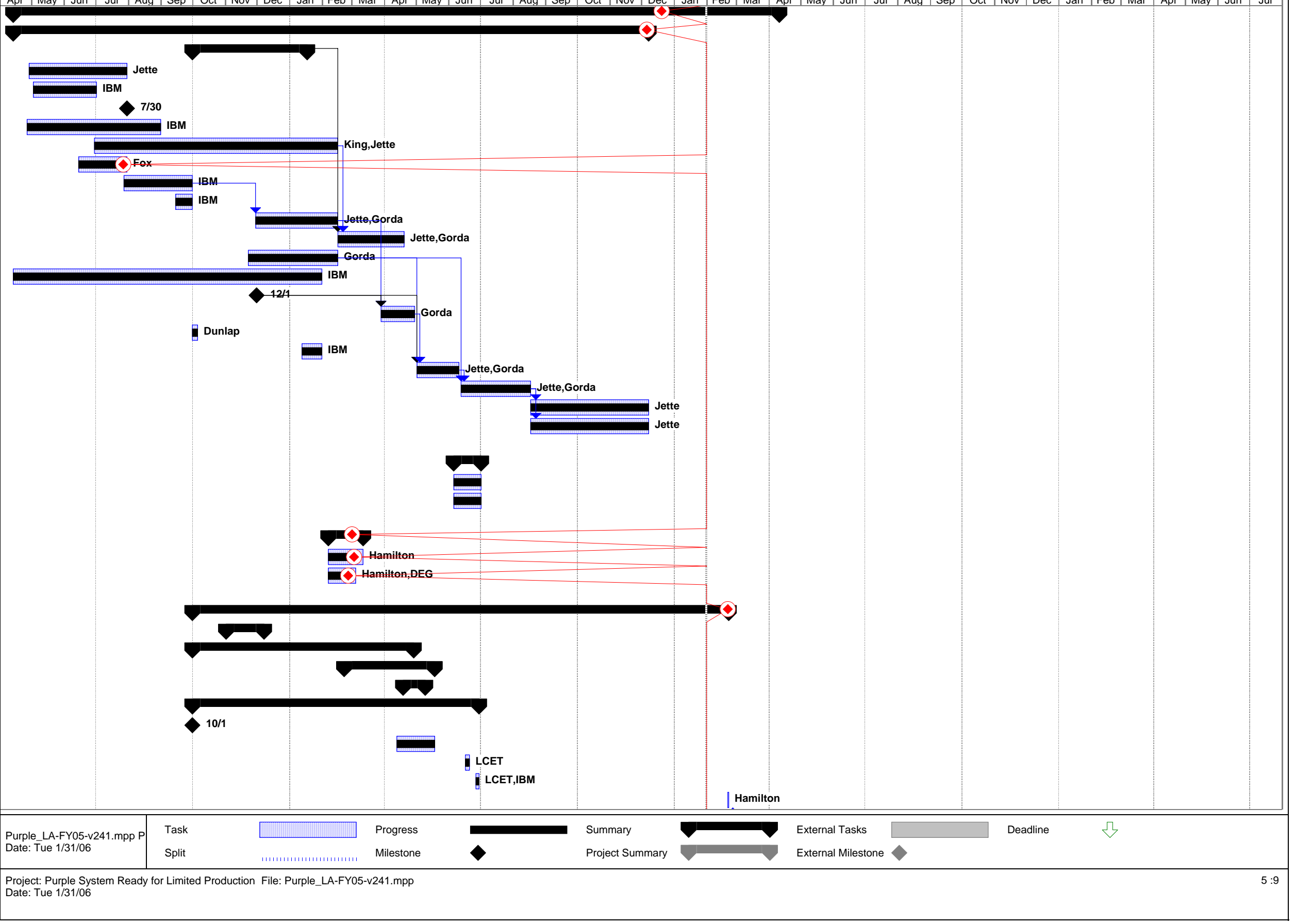


Purple Integration Project

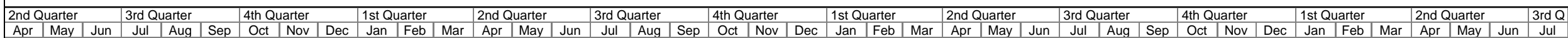

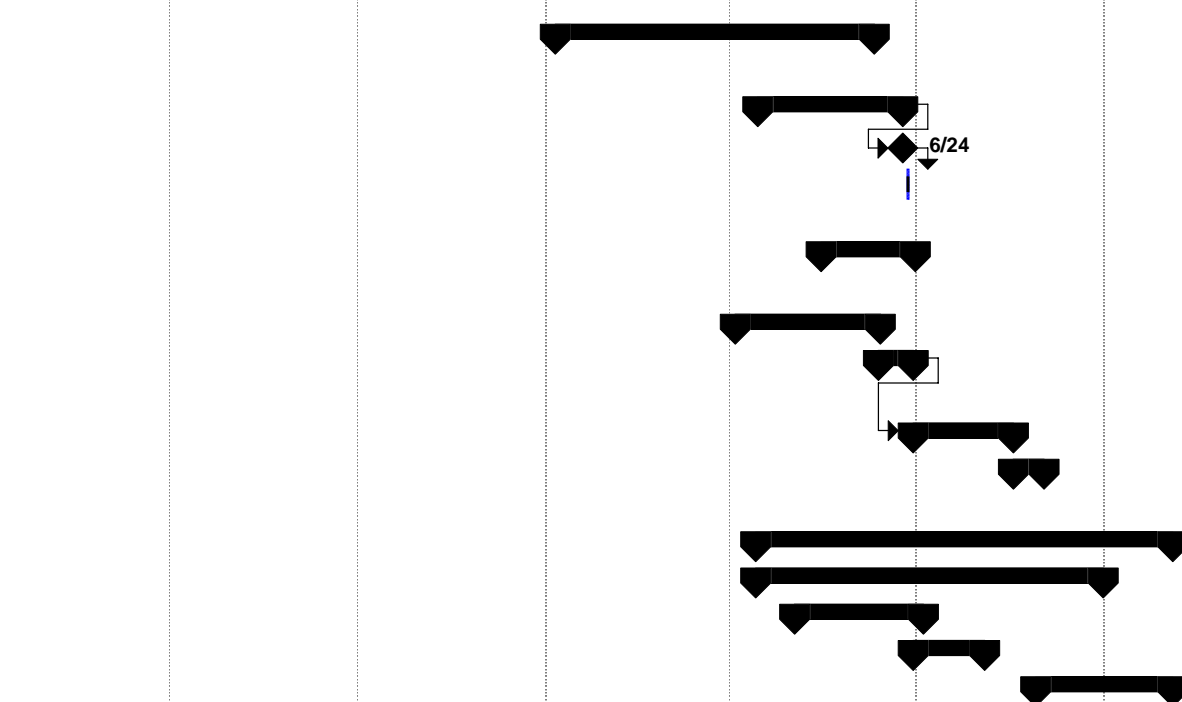

Loewe,Fox,Hadjimarkos,IBM

Loewe,Fox,Hadjimarkos,IBM

$\rightarrow 11 / 18$

四

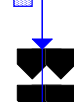

1

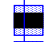

$=$

Tomasenke

Springrneye

Wolfe

Gyllenraal

Chambeau

-

5

\begin{tabular}{|l|ll} 
Purple_LA-FY05-v241.mpp P & Task $\quad \square$ Progress \\
\hline
\end{tabular}

Milestone

Summary

External Tasks

External Milestone

Deadline

Project: Purple System Ready for Limited Production File: Purple_LA-FY05-v241.mpp

Project Summary

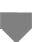




\section{Purple Integration Project}

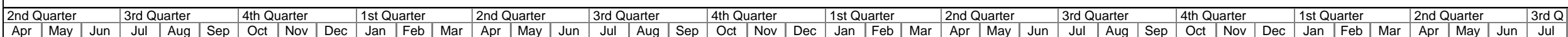

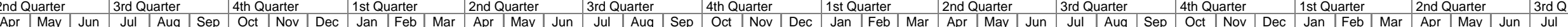

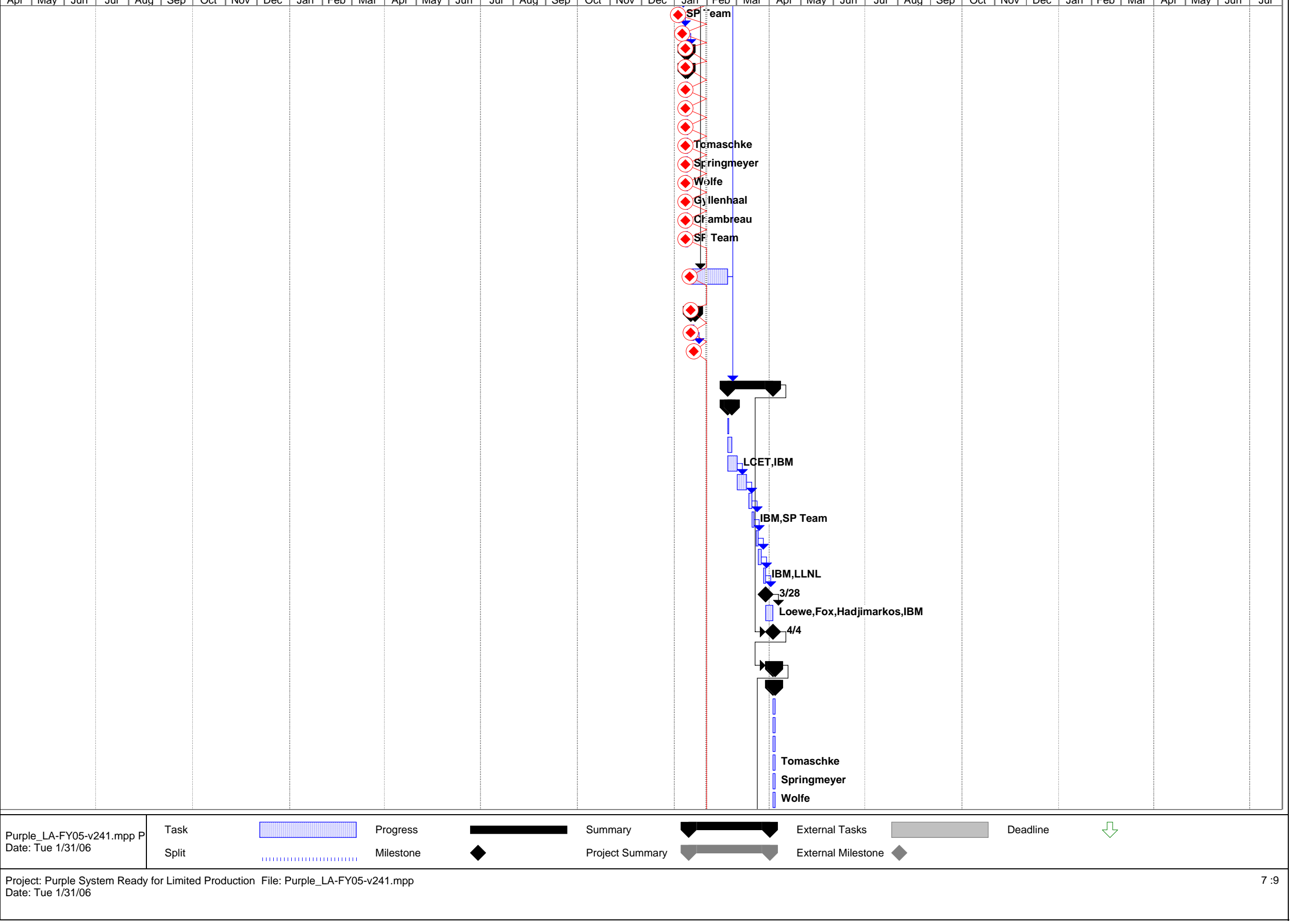




\section{Purple Integration Project}

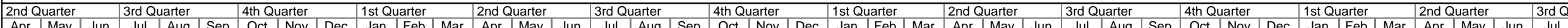
Apr May Ju Jul Aug Se Oct Nov De Jan Feb Ma Ap May Jun Jul Aug Sep Oct Nov Dec Jan Feb Mar Apr May

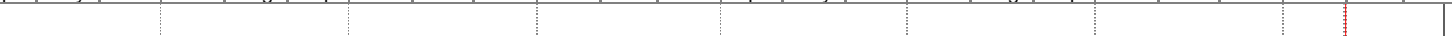

Chambreau

SP Team

DSG,SP Team 


\section{Purple Integration Project}

208 Initial Purple (1280) System Acceptance Complete

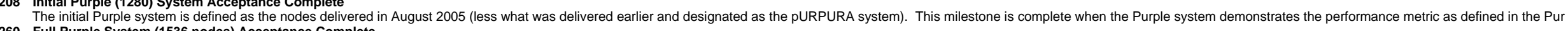

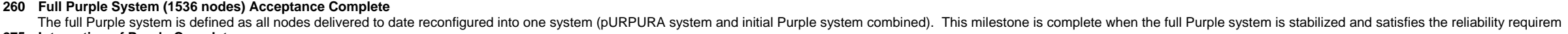

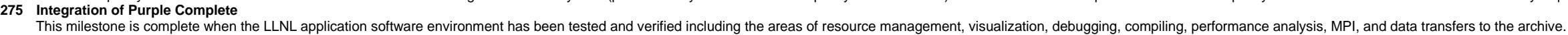

